\title{
Novel Synthesis of Heterocycles Having a Functionalized Carbon Center via Nickel-Mediated Carboxylation: Total Synthesis of Erythrocaline
}

\author{
Kazuya Shimizu, Masanori Takimoto and Miwako Mori* \\ Graduate School of Pharmaceutical Sciences, Hokkaido University, \\ Sapporo 060-0812, Japan
}

\section{Supporting Information}

General Information. ${ }^{1} \mathrm{H}$ NMR and ${ }^{13} \mathrm{C}$ NMR were recorded on a JEOL EX-270 (270 MHz for ${ }^{1} \mathrm{H}, 67.5 \mathrm{MHz}$ for ${ }^{13} \mathrm{C}$ ), or JEOL AL-400 (400 MHz for ${ }^{1} \mathrm{H}, 100 \mathrm{MHz}$, for ${ }^{13} \mathrm{C}$ ) instrument in $\mathrm{CDCl}_{3}$ with tetramethylsilane as an internal standard otherwise mentioned. Data are reported as follows: chemical shift in ppm $(d)$, multiplicity ( $\mathrm{s}=$ singlet, $\mathrm{d}=$ doublet, $\mathrm{t}=$ triplet, $\mathrm{q}=$ quartet, $\mathrm{m}=$ multiplet, $\mathrm{br}=$ broad signal), coupling constant $(\mathrm{Hz})$, integration. Infrared spectra (IR) were obtained on a Perkin Elmer 1605 FTIR spectrometer. Mass spectra were obtained on either a JEOL JMSFABmate (EI), a JEOL JMS-HX110 (FAB), or a JEOL JMS-700TZ (ESI). Silica gel column chromatography was performed by Merck Silica Gel 60 (70-230 or 230-400 mesh ATM). For analytical or preparative TLC, Merck Silica Gel $60 \mathrm{PF}_{254}$ was used. All solvents and reagents were purified when necessary using standard procedures. $\mathrm{Ni}(\mathrm{cod})_{2}$ was prepared by a literature procedure ${ }^{1}$ and handled under an argon atmosphere. All reactions were carried out under argon. $\mathrm{Me}_{2} \mathrm{Zn}$ was purchased from Kanto Chemical Co. Inc. Alkynyl zinc reagent was prepared from lithium TNS acetylide and $\mathrm{ZnCl}_{2}$ in the usual method.

Typical Procedure for the synthesis of $\alpha, \beta$-unsaturated ester (2a) To a stirred suspension of $\mathrm{Ni}(\mathrm{cod})_{2}(110 \mathrm{mg}, 0.40 \mathrm{mmol})$ and $\mathrm{DBU}(0.12 \mathrm{~mL}, 0.80 \mathrm{mmol})$ in degassed THF (5.8 mL) was slowly added $1 \mathrm{a}(89 \mathrm{mg}, 0.36 \mathrm{mmol})$ at $0{ }^{\circ} \mathrm{C}$ for $1 \mathrm{~h}$ and 
the solution was stirred at the same temperature for $2 \mathrm{~h}$. To this solution was added $\mathrm{Me}_{2} \mathrm{Zn}(1.0 \mathrm{M}$ hexane solution_1.1 mL, $1.1 \mathrm{mmol})$ at $0{ }^{\circ} \mathrm{C}$ and the solution was stirred at $0{ }^{\circ} \mathrm{C}$ until the spot of 1 a disappeared on TLC. To this solution was added $10 \% \mathrm{HCl}$ and the aqueous layer was extracted with ethyl acetate. The combined organic layer was washed with brine, dried over $\mathrm{Na}_{2} \mathrm{SO}_{4}$, and concentrated. The crude product was treated with $\mathrm{CH}_{2} \mathrm{~N}_{2}$ and it was purified by column chromatography (hexane/ethyl acetate, 10/1) on silica gel to give a crude $\alpha, \beta$-unsaturated ester 2a (94 $\mathrm{mg}, 81 \%)$

Typical Procedure for Synthesis of Heterocycles Using Michael Addition. To a solution of $2 \mathrm{a}(53.4 \mathrm{mg}, 0.167 \mathrm{mmol})$ in THF $(1.0 \mathrm{ml})$ was added TBAF $(1.0 \mathrm{M}$ THFsolution, $0.4 \mathrm{~mL}, 0.4 \mathrm{mmol}$ ) at $0_{\text {_ }}$ and the solution was stirred at $0{ }^{\circ} \mathrm{C}$ for 100 min. To this solution was added aqueous sat. $\mathrm{NH}_{4} \mathrm{Cl}$ solution and the aqueous layer was extracted with ethyl acetate. The organic layer was washed with brine, dried over $\mathrm{Na}_{2} \mathrm{SO}_{4}$ and concentrated. The residue was purified by column chromatography on silica gel (hexane/ethyl acetate, 3/1) to give isobenzofurane $\mathbf{3 a}$ (28 mg, 81\%).

\section{Substrate for Synthesis of Heterocycles}

tert-Butyl-(2-ethynyl-benzyloxy)-dimethylsilane (1a). IR (neat) 3301, 2955, 2100, $1078 \mathrm{~cm}^{-1} ;{ }^{1} \mathrm{H}$ NMR $\left(400 \mathrm{MHz}, \mathrm{CDCl}_{3}\right) d 0.15(\mathrm{~s}, 6 \mathrm{H}), 1.00(\mathrm{~s}, 9 \mathrm{H}), 3.31(\mathrm{~s}, 1 \mathrm{H})$, 4.94 (s, 2 H), 7.21 (dd, $J=8.0,8.0 \mathrm{~Hz}, 1 \mathrm{H}), 7.39$ (dd, $J=8.0,8.0 \mathrm{~Hz}, 1 \mathrm{H}), 7.47$ (d, $J=8.0 \mathrm{~Hz}, 1 \mathrm{H}), 7.59(\mathrm{~d}, J=8.0 \mathrm{~Hz}, 1 \mathrm{H}) ;{ }^{13} \mathrm{C} \mathrm{NMR}(100 \mathrm{MHz}$, $\left.\mathrm{CDCl}_{3}\right)_{-} \delta-5.22,18.47,26.02,63.10,81.03,82.00,118.73,125.69,126.33,128.89,1$

32.20, 143.72; LR MS (ESI) m/z $246\left(\mathrm{M}^{+}\right), 231,189,115,75$; HR MS (EI) calcd for $\mathrm{C}_{15} \mathrm{H}_{22} \mathrm{OSi}$ 246.1440, found 246.1456.

tert-Butyl-[2-(2-ethynyl-phenyl)-ethoxy]-dimethyl-silane (1b). IR (neat) 3303, 2105, $1094 \mathrm{~cm}^{-1} ;{ }^{1} \mathrm{H}$ NMR (400 MHz, $\left.\mathrm{CDCl}_{3}\right) \delta 0.00(\mathrm{~s}, 6 \mathrm{H}), 0.89(\mathrm{~s}, 9 \mathrm{H}), 3.05(\mathrm{t}, J$ $=7.6 \mathrm{~Hz}, 2 \mathrm{H}), 3.25(\mathrm{~s}, 1 \mathrm{H}), 3.87(\mathrm{t}, J=7.6 \mathrm{~Hz}, 2 \mathrm{H}), 7.13-7.30(\mathrm{~m}, 3 \mathrm{H}), 7.47(\mathrm{~d}, J=$ 
$7.6 \mathrm{~Hz}, 1 \mathrm{H}) ;{ }^{13} \mathrm{C}$ NMR $\left(100 \mathrm{MHz}, \mathrm{CDCl}_{3}\right) \delta-5.31,18.39,25.99,38.17,63.31,80.53$, 82.30, 121.75, 126.05, 128.56, 130.00, 132.73, 141.48; LR MS (EI) $\mathrm{m} / z 260\left(\mathrm{M}^{+}\right)$, 245, 203, 129, 115, 75; HR MS (EI) calcd for $\mathrm{C}_{16} \mathrm{H}_{24} \mathrm{OSi} 260.1596$, found 260.1602.

Benzyl-(2-ethynyl-benzyl)-carbamic acid tert-butyl ester (1c). IR (neat) 3291, 2976, 1694, 1165, $760 \mathrm{~cm}^{-1}$; ${ }^{1} \mathrm{H}$ NMR (400 MHz, $\left.\mathrm{CDCl}_{3}\right) \delta 1.45$ (s, 9/2 H), 1.48 (s, 9/2 H), 3.20 (s, $1 \mathrm{H}), 4.36$ (s, 2/2 H), 4.47 (s, 2/2 H), 4.56 (s, 2/2 H), 4.76 (s, 2/2 H), 7.19-7.48 (m, $9 \mathrm{H}$ ); ${ }^{13} \mathrm{C}$ NMR (100 MHz, $\mathrm{CDCl}_{3}$ ) $\delta 28.41,47.66$ (Bn-C), 48.05 (BnC), 49.93, 80.03, 81.31, 81.90 (alkyne-C), 82.15 (alkyne-C), 126.23, 126.67 (Ar-CH), 126.84 (Ar-CH), 127.07, 127.34 (Ar-CH), 127.50 (Ar-CH), 128.29, 129.01, 132.76, 137.86 (Ar-C), 138.10 (Ar-C), 140.35 (Ar-C), 155.91 (Ar-C); LR MS (EI) m/z 265 $\left(\mathrm{M}^{+}-{ }^{-} \mathrm{Bu}-\mathrm{H}\right), 248,220,115$; HR MS (EI) calcd for $\mathrm{C}_{17} \mathrm{H}_{14} \mathrm{O}_{2} \mathrm{~N}$ (-Bu) 264.1024, found 264.1022 .

Benzyl-[2-(2-ethynyl-phenyl)-ethyl]-carbamic acid tert-butyl ester (1d). ${ }^{1} \mathrm{H}$ NMR (400 MHz, $\mathrm{CDCl}_{3}$ ) $\delta 1.45$ (s, 9 H), 2.99 (br, 17/15 H), ), 3.07 (br, 13/15 H), 3.21 (s, $1 \mathrm{H}), 3.41$ (br, $2 \mathrm{H}), 4.32$ (s, 13/15 H), 4.43 (br, 17/15 H), 7.12-7.48 (m, 8 H), $3.07(\mathrm{~d}, J=7.6 \mathrm{~Hz}, 1 \mathrm{H}) ;{ }^{13} \mathrm{C} \mathrm{NMR}\left(100 \mathrm{MHz}, \mathrm{CDCl}_{3}\right) \delta 27.45,28.45,33.21,47.17$, $50.40,79.75,80.64,82.09,121.67,126.19,127.02,127.15,127.76,128.33,128.92$, 129.46, 132.84, 138.35, 138.65; LR MS (EI) $m / z 335\left(\mathrm{M}^{+}\right), 262,234,220,91,57$; HR MS (EI) calcd for $\mathrm{C}_{22} \mathrm{H}_{25} \mathrm{NO}_{2} 335.1885$, found 335.1885.

\section{Synthesis of Heterocycles}

3-[2-(tert-Butyl-dimethyl-silanyloxymethyl)-phenyl]-but-2-enoic acid methyl ester (2a). IR (neat) 2952, 1721, 1641, 1169, $838 \mathrm{~cm}^{-1} ;{ }^{1} \mathrm{H}$ NMR (400 MHz, $\mathrm{CDCl}_{3}$ ) $\delta$ 0.08 (s, $6 \mathrm{H}), 0.92$ (s, 9 H), 2.46 (s, 3 H), 3.74 (s, 3 H), 4.65 (s, 2 H), 5.80 (s, 1 H), $7.07(\mathrm{~d}, J=7.2 \mathrm{~Hz}, 1 \mathrm{H}), 7.24(\mathrm{dd}, J=7.6,7.0 \mathrm{~Hz}, 1 \mathrm{H}), 7.31(\mathrm{dd}, J=7.2,7.0 \mathrm{~Hz}, 1$ H), $7.50(\mathrm{~d}, J=7.2,7.0 \mathrm{~Hz}, 1 \mathrm{H}) ;{ }^{13} \mathrm{C} \mathrm{NMR}\left(100 \mathrm{MHz}, \mathrm{CDCl}_{3}\right) \delta-5.27,18.38,21.07$, 
25.94, 51.03, 62.65, 118.99, 126.84, 126.92, 127.61, 127.75, 136.99, 142.11, 157.20, 166.70; LR MS (EI) m/z 315 (M+Me), 289, 263, 59; HR MS (EI) calcd for $\mathrm{C}_{14} \mathrm{H}_{19} \mathrm{O}_{3} \mathrm{Si}$ (M-Me) 263.1103, found 263.1115.

(1-Methyl-1,3-dihydro-isobenzofuran-1-yl)-acetic acid methyl ester (3a). IR (neat) $1738,1030 \mathrm{~cm}^{-1} ;{ }^{1} \mathrm{H}$ NMR (400 MHz, $\mathrm{CDCl}_{3}$ ) $\delta 1.62$ (s, $3 \mathrm{H}$ ), 2.77 (d, $J=14.4$ $\mathrm{Hz}, 1 \mathrm{H}), 2.82(\mathrm{~d}, J=14.4 \mathrm{~Hz}, 1 \mathrm{H}), 3.60(\mathrm{~s}, 3 \mathrm{H}), 5.08(\mathrm{~d}, J=12.4 \mathrm{~Hz}, 1 \mathrm{H}), 5.11(\mathrm{~d}$, $J=12.4 \mathrm{~Hz}, 1 \mathrm{H}), 7.14-7.29(\mathrm{~m}, 4 \mathrm{H}) ;{ }^{13} \mathrm{C}$ NMR $\left(100 \mathrm{MHz}, \mathrm{CDCl}_{3}\right) \delta 27.12,45.80$, $51.51,71.62,85.91,120.90,120.95,127.28,127.64,138.55,144.30,170.48$; LR MS (EI) $\mathrm{m} / \mathrm{z} 206\left(\mathrm{M}^{+}\right), 191,133,91,77$; HR MS (EI) calcd for $\mathrm{C}_{12} \mathrm{H}_{14} \mathrm{O}_{3} 206.0939$, found 206.0943; Anal. Calcd for $\mathrm{C}_{12} \mathrm{H}_{14} \mathrm{O}_{3}$ : C, 69.88; H, 6.84. Found: C, 69.83; H, 6.95.

3-\{2-[2-(tert-Butyl-dimethyl-silanyloxy)-ethyl]-phenyl\}-but-2-enoic acid methyl ester (2b). IR (neat) 2952, 1721, 1640, 1169, 1094, $838 \mathrm{~cm}^{-1}$; ${ }^{1} \mathrm{H}$ NMR $(400 \mathrm{MHz}$, $\left.\mathrm{CDCl}_{3}\right) \delta-0.01(\mathrm{~s}, 6 \mathrm{H}), 0.86(\mathrm{~s}, 9 \mathrm{H}), 2.47(\mathrm{~d}, J=1.2 \mathrm{~Hz}, 3 \mathrm{H}), 2.81(\mathrm{t}, J=7.2 \mathrm{~Hz}, 2$ H), $3.74(\mathrm{~s}, 3 \mathrm{H}), 3.75$ (t, $J=7.2 \mathrm{~Hz}, 2 \mathrm{H}), 5.78(\mathrm{q}, 1 \mathrm{H}), 7.04-7.26(\mathrm{~m}, 4 \mathrm{H}) ;{ }^{13} \mathrm{C}$ NMR (100 MHz, $\left.\mathrm{CDCl}_{3}\right) \delta-5.33,18.42,21.71,25.97,51.06,64.27,119.23,126.11$, 127.12, 127.51, 130.19, 134.65, 143.99, 158.14, 166.71; LR MS (EI) m/z $319\left(\mathrm{M}^{+}-\right.$ Me), 303, 277, 115, 59; HR MS (EI) calcd for $\mathrm{C}_{19} \mathrm{H}_{30} \mathrm{O}_{3} \mathrm{Si}$ (M-Me) 334.1729, found 319.1733; Anal. Calcd for $\mathrm{C}_{12} \mathrm{H}_{14} \mathrm{O}_{3}$ : C, 68.22; H, 9.04. Found: C, 68.12; H, 8.90.

(1-Methyl-isochroman-1-yl)-acetic acid methyl ester (3b). IR (neat) 2949, 1737, $1099 \mathrm{~cm}^{-1} ;{ }^{1} \mathrm{H}$ NMR (400 MHz, $\left.\mathrm{CDCl}_{3}\right) \delta 1.63$ (s, $\left.3 \mathrm{H}\right), 2.77(\mathrm{~d}, J=14.0 \mathrm{~Hz}, 1 \mathrm{H})$, 2.77-2.89 (m, 2 H), 2.93 (d, $J=14.0 \mathrm{~Hz}, 1 \mathrm{H}), 3.63$ (s, $3 \mathrm{H}), 3.90-4.04$ (m, 2 H), 7.05$7.20(\mathrm{~m}, 4 \mathrm{H}) ;{ }^{13} \mathrm{C}$ NMR $\left(100 \mathrm{MHz}, \mathrm{CDCl}_{3}\right) \delta 27.78,29.24,46.49,51.53,59.87$, 75.19, 125.05, 126.19, 126.32, 128.84, 133.18, 140.86, 170.47; LR MS (EI) $\mathrm{m} / \mathrm{z}, 220$ $\left(\mathrm{M}^{+}\right), 205,147,91,77$; HR MS (EI) calcd for $\mathrm{C}_{13} \mathrm{H}_{16} \mathrm{O}_{3}$ 220.1084, found 220.1099; Anal. Calcd for $\mathrm{C}_{13} \mathrm{H}_{16} \mathrm{O}_{3}$ : C, 70.89; H, 7.32. Found: C, 70.70; H, 7.42. 
3-\{2-[(Benzyl-tert-butoxycarbonyl-amino)-methyl]-phenyl\}-but-2-enoic acid methyl ester (2c). ${ }^{1} \mathrm{H}$ NMR (400 MHz, $\left.\mathrm{CDCl}_{3}\right) \delta 1.47$ (s, $9 \mathrm{H}$ ), 2.35 (s, $3 \mathrm{H}$ ), 3.70 (s, $3 \mathrm{H}), 4.34-4.46(\mathrm{~m}, 4 \mathrm{H}), 5.69$ (s, $1 \mathrm{H}), 7.05-7.32(\mathrm{~m}, 9 \mathrm{H}) ;{ }^{13} \mathrm{C} \mathrm{NMR}(100 \mathrm{MHz}$, $\left.\mathrm{CDCl}_{3}\right) \delta 21.71,29.05,47.37,50.02,51.67,80.77,119.84,127.62,127.81,127.88$, 128.43, 128.54, 129.05, 143.30, 138.24, 143.89, 156.47, 157.65, 167.03; LR MS (EI) m/z $395\left(\mathrm{M}^{+}\right), 339,294,91$; HR MS (EI) calcd for $\mathrm{C}_{24} \mathrm{H}_{29} \mathrm{O}_{4} \mathrm{~N}$ 395.2096, found 395.2097.

(2-Benzyl-1-methyl-2,3-dihydro-1 $H$-isoindol-1-yl)-acetic acid methyl ester (3c). IR (neat) 2949, 1732, 1209, $1170 \mathrm{~cm}^{-1} ;{ }^{1} \mathrm{H}$ NMR (400 MHz, $\left.\mathrm{CDCl}_{3}\right) \delta 1.52(\mathrm{~s}, 3 \mathrm{H})$, $2.80(\mathrm{~d}, J=13.5 \mathrm{~Hz}, 1 \mathrm{H}), 2.86$ (d, $J=13.5 \mathrm{~Hz}, 1 \mathrm{H}), 3.55$ (s, $3 \mathrm{H}), 3.74$ (d, $J=12.9$ $\mathrm{Hz}, 1 \mathrm{H}$ ), 3.84 ( d, $J=13.0 \mathrm{~Hz}, 1 \mathrm{H}$ ), 3.88 ( d, $J=13.0 \mathrm{~Hz}, 1 \mathrm{H}$ ), 3.99 ( d, $J=12.9$ $\mathrm{Hz}, 1 \mathrm{H}), 7.13-7.44(\mathrm{~m}, 9 \mathrm{H}) ;{ }^{13} \mathrm{C} \mathrm{NMR}\left(100 \mathrm{MHz}, \mathrm{CDCl}_{3}\right) \delta 23.25,42.52,51.28$, $52.22,55.44,67.22,122.22,122.82,126.51,126.82,126.89,128.20,128.41,138.28$, 139.61, 146.42, 171.47; LR MS (EI) $\mathrm{m} / z 295\left(\mathrm{M}^{+}\right), 280,222,204,91$; HR MS (EI) calcd for $\mathrm{C}_{19} \mathrm{H}_{21} \mathrm{NO}_{2} 295.1572$, found 295.1574 .

3-\{2-[2-(Benzyl-tert-butoxycarbonyl-amino)-ethyl]-phenyl\}-but-2-enoic acid methyl ester (2d). ${ }^{1} \mathrm{H}$ NMR (400 MHz, $\left.\mathrm{CDCl}_{3}\right) \delta 1.47$ (s, $\left.9 \mathrm{H}\right), 2.41$ (s, $3 \mathrm{H}$ ), 2.75 (br, 2 H), 3.26 (s, 8/7 H), 3.36 (s, 6/7 H), 3.75 (s, 3 H), 4.30 (s, 6/7 H), 4.39 (s, 8/7 H), $5.74(\mathrm{~s}, 1 \mathrm{H}), 7.01-7.31(\mathrm{~m}, 9 \mathrm{H}) ;{ }^{13} \mathrm{C}$ NMR $\left(100 \mathrm{MHz}, \mathrm{CDCl}_{3}\right) \delta 21.62$, 28.41, 31.51, 48.20, 50.03, 51.01, 79.70, 119.12, 126.17, 127.07, 127.19, 127.77, $128.34,129.85,134.94,138.12,143.71,155.37,157.85,166.49$.

(2-Benzyl-1-methyl-1,2,3,4-tetrahydro-isoquinolin-1-yl)-acetic acid methyl ester (3d). ${ }^{1} \mathrm{H}$ NMR (400 MHz, $\mathrm{CDCl}_{3}$ ) $\delta 1.56$ (s, $3 \mathrm{H}$ ), 2.56-2.66 (m, $2 \mathrm{H}$ ), 2.74-2.84 (m, 2 H), $2.96(\mathrm{~d}, J=14.0 \mathrm{~Hz}, 1 \mathrm{H}), 3.03(\mathrm{~d}, J=14.0 \mathrm{~Hz}, 1 \mathrm{H}), 3.50(\mathrm{~s}, 1 \mathrm{H}), 3.56(\mathrm{~d}, J=$ 
$14.0 \mathrm{~Hz}, 1 \mathrm{H}), 4.08(\mathrm{~d}, J=14.0 \mathrm{~Hz}, 1 \mathrm{H})$ 7.04-7.56 (m, $9 \mathrm{H}) ;{ }^{13} \mathrm{C}$ NMR $(100 \mathrm{MHz}$, $\left.\mathrm{CDCl}_{3}\right) \delta 23.82,29.91,42.81,45.29,51.17,53.33,60.30,125.61,125.70,126.44,126$ $.60,128.11,128.63,135.45,140.42,141.97,171.20$.

3-\{2-[(Benzyl-tert-butoxycarbonyl-amino)-methyl]-phenyl $\}-5-($ trimethyl-silanyl)pent-2-en-4-ynoic acid methyl ester (2e). ${ }^{1} \mathrm{H}$ NMR $\left(270 \mathrm{MHz}, \mathrm{CDCl}_{3}\right) \delta 0.15(\mathrm{~s}, 9$ H), 1.43 (s, 9 H), 3.78 (s, 3 H), 4.45 (br, 4 H), 6.05 (s, 1 H), 7.20-7.37 (m, 10 H).

[2-Benzyl-1-(trimethyl-silanylethynyl)-2,3-dihydro-1H-isoindol-1-yl]-acetic acid methyl ester (3e). ${ }^{1} \mathrm{H}$ NMR $\left(270 \mathrm{MHz}, \mathrm{CDCl}_{3}\right) \delta 0.16$ (s, $\left.9 \mathrm{H}\right), 3.13$ (s, $\left.2 \mathrm{H}\right), 3.56$ (s, $3 \mathrm{H}), 3.63(\mathrm{~d}, J=13.2 \mathrm{~Hz}, 1 \mathrm{H}), 3.68(\mathrm{~d}, J=13.2 \mathrm{~Hz}, 1 \mathrm{H}), 3.90(\mathrm{~d}, J=13.2 \mathrm{~Hz}, 1$ $\mathrm{H}), 4.18(\mathrm{~d}, J=13.2 \mathrm{~Hz}, 1 \mathrm{H})$.

\section{Total Synthesis of Erythrocaline}

Trimethyl-[6-(2-nitro-vinyl)-benzo[1,3]dioxol-5-ylethynyl]-silane (10). A solution of 9 (246 mg, $1.0 \mathrm{mmol}), \mathrm{NH}_{4} \mathrm{OAc}(64 \mathrm{mg}, 0.83 \mathrm{mmol})$ and $\mathrm{CH}_{3} \mathrm{NO}_{2}(0.27 \mathrm{ml}, 5.0$ $\mathrm{mmol})$ in $\mathrm{AcOH}(2.5 \mathrm{ml})$ was heated at $100{ }^{\circ} \mathrm{C}$ for $7 \mathrm{~h}$. Solvent was removed and the residue was purified by recrystalization from ether to give colorless crystals of $\mathbf{1 0}$ (1.23 g, $85 \%$ ). IR (film) 3104, 2955, 2149, 1610, 1330, 1253, $1034 \mathrm{~cm}^{-1}$; ${ }^{1} \mathrm{H}$ NMR $\left(400 \mathrm{MHz}, \mathrm{CDCl}_{3}\right) \delta 0.28(\mathrm{~s}, 9 \mathrm{H}), 6.05(\mathrm{~s}, 2 \mathrm{H}), 6.94(\mathrm{~s}, 1 \mathrm{H}), 6.96(\mathrm{~s}, 1 \mathrm{H}), 7.57$ (d, $J=13.6 \mathrm{~Hz}, 1 \mathrm{H}), 8.46(\mathrm{~d}, J=13.6 \mathrm{~Hz}, 1 \mathrm{H}) ;{ }^{13} \mathrm{C} \mathrm{NMR}\left(100 \mathrm{MHz}, \mathrm{CDCl}_{3}\right) \delta-0.21$, $101.35,101.40,101.90,105.95,112.34,120.84,126.62,136.50,136.92,148.63$, 150.50; LR MS (EI) $m / z 289\left(\mathrm{M}^{+}\right), 243,73$; HR MS (EI) calcd for $\mathrm{C}_{14} \mathrm{H}_{15} \mathrm{NO}_{4} \mathrm{Si}$ 289.0770, found 289.0788; Anal. Calcd for $\mathrm{C}_{14} \mathrm{H}_{15} \mathrm{NO}_{4} \mathrm{Si}$ : C, 58.11; H, 5.22; N, 4.84. Found: C, 58.06; H, 5.32; N, 4.82 . 
[2-(6-Ethynyl-benzo[1,3]dioxol-5-yl)-ethyl]-carbamic acid tert-butyl ester (1f). To a suspension of $\mathrm{LiAlH}_{4}(885 \mathrm{mg}, 23.3 \mathrm{mmol})$ in ether $(25 \mathrm{ml})$ was added $10(2.25 \mathrm{~g})$ in ether $(25 \mathrm{ml})$ at $-78_{-}$and the solution was stirred at room temperature for $3 \mathrm{~h}$. Water $(0.9 \mathrm{ml}), 15 \%$ aqueous $\mathrm{NaOH}$ solution $(0.9 \mathrm{ml})$ and water $(2.7 \mathrm{ml})$ were added to this solution at $-78{ }^{\circ} \mathrm{C}$ and the solution was stirred at room temperature for $14 \mathrm{~h}$. An undissolved material was filtered off and the filtrate was concentrated. The residue was dissolved in $\mathrm{MeOH}(26 \mathrm{ml})$ and to this solution was added $\mathrm{NEt}_{3}(1.6 \mathrm{ml}$, $11.66 \mathrm{mmol})$ and $(\mathrm{Boc})_{2} \mathrm{O}(2.7 \mathrm{ml}, 11.66 \mathrm{mmol})$. The solution was stirred at room temperature for $14 \mathrm{~h}$. After solvent was removed, the residue was purified by column chromatography on silica gel (hexane/ethyl acetate, 10/1) to give colorless oil of $\mathbf{1 f}$ (1.37 g, 61\%). IR (neat) 3290, 2977, 2101, 1700, 1366, 1252, $1038 \mathrm{~cm}^{-1}$; ${ }^{1} \mathrm{H}$ NMR $\left(400 \mathrm{MHz}, \mathrm{CDCl}_{3}\right) \delta 1.43(\mathrm{~s}, 9 \mathrm{H}), 2.92(\mathrm{t}, J=6.8 \mathrm{~Hz}, 2 \mathrm{H}), 3.37(\mathrm{td}, J=6.8,6.4 \mathrm{~Hz}$, $2 \mathrm{H}), 4.57$ (s, $1 \mathrm{H}), 5.96$ (s, $2 \mathrm{H}), 6.91(\mathrm{~s}, 1 \mathrm{H}), 7.26$ (s, $1 \mathrm{H}) ;{ }^{13} \mathrm{C}$ NMR $(100 \mathrm{MHz}$, $\left.\mathrm{CDCl}_{3}\right) \delta 28.43,34.60,41.08,79.11,79.53,82.10,101.32,109.55,112.14,114.39,13$

6.72, 145.78, 148.29, 155.71; LR MS (EI) $m / z 289\left(\mathrm{M}^{+}\right), 233,216,188,172,159,57$; HR MS (EI) calcd for $\mathrm{C}_{16} \mathrm{H}_{19} \mathrm{NO}_{4}$ 289.1314, found 289.1315; Anal. Calcd for $\mathrm{C}_{16} \mathrm{H}_{19} \mathrm{NO}_{4}$ : C, 66.42; H, 6.62; N, 4.84. Found: C, 66.23; H, 6.61; N, 4.74.

\section{_3-[6-(2-tert-Butoxycarbonylamino-ethyl)-benzo[1,3]dioxol-5-yl]-5-(trimethyl-}

silanyl)-pent-2-en-4-ynoic acid methyl ester (2f). According to the typical procedure for the synthesis of $\alpha, \beta$-unsaturated ester, $\alpha, \beta$-unsaturated acid was synthesized from $\mathrm{Ni}(\mathrm{cod})_{2}(109 \mathrm{mg}, 0.4 \mathrm{mmol}), \mathrm{DBU}(0.18 \mathrm{~mL}, 1.2 \mathrm{mmol}), \mathbf{1 f}(0.36$ mmol) and alkynyl zinc reagent $5(2.2 \mathrm{~mL}, 1.1 \mathrm{mmol})$ in THF $(5.8 \mathrm{~mL})$. The crude product was converted into ester $\mathbf{2 f}$, which was purified by column chromatography on silica gel (hexane/ethyl acetate, 5/1) to give $\mathbf{2 f}(111.2 \mathrm{mg}, 69 \%)$. 
methyl ester (3f). A solution of $\mathrm{CH}_{2} \mathrm{Cl}_{2}(8.4 \mathrm{ml})$ of $\mathbf{2 f}(933 \mathrm{mg}, 3.1 \mathrm{mmol})$ and $\mathrm{CF}_{3} \mathrm{CO}_{2} \mathrm{H}$ (1.6 mL, $21 \mathrm{mmol}, 10$ equiv.) was stirred at room temperature for $3 \mathrm{~h}$. After solvent was removed, the residue was dissolved in ethyl acetate. The organic layer was washed with sat. $\mathrm{NaHCO}_{3}$ solution and brine, and dried over $\mathrm{Na}_{2} \mathrm{SO}_{4}$ and concentrated. The residue was dissolved in $\mathrm{MeOH}(20 \mathrm{ml})$ and the solution was refluxed for $18 \mathrm{~h}$. Solvent was removed and the residue was dissolved in THF (8 ml). To this solution was added TBAF (THF solution, 1.0 M, $2.3 \mathrm{~mL}, 1.1$ equiv.) and the solution was stirred at room temperature for $1 \mathrm{~h}$. Water was added and the aqueous layer was extracted with ethyl acetate and the organic layer was washed with sat.. $\mathrm{NaHCO}_{3}$ solution and brine, and dried over $\mathrm{Na}_{2} \mathrm{SO}_{4}$ and concentrated. The residue was purified by column chromatography on silica gel (ethyl acetate) to give $\mathbf{3 f}$ (438 mg, 76\%). IR (film) 3286, 2952, $1734 \mathrm{~cm}^{-1} ;{ }^{1} \mathrm{H}$ NMR (400 MHz, $\mathrm{CDCl}_{3}$ ) $\delta 2.46$ (s, 1 H), 2.62 (ddd, $J=16.0,4.0,3.2 \mathrm{~Hz}, 1 \mathrm{H}), 2.84$ (ddd, $J=16.0,10.4,5.6 \mathrm{~Hz}, 1 \mathrm{H}$ ), $2.89(\mathrm{~d}, J=16.0 \mathrm{~Hz}, 1 \mathrm{H}), 3.12(\mathrm{ddd}, J=12.0,5.6,3.2 \mathrm{~Hz}, 1 \mathrm{H}), 3.13(\mathrm{~d}, J=16.0 \mathrm{~Hz}$, $1 \mathrm{H}), 3.22$ (ddd, $J=12.0,10.4,4.0 \mathrm{~Hz}, 1 \mathrm{H}), 3.70$ (s, $3 \mathrm{H}), 5.90(\mathrm{~d}, J=1.2 \mathrm{~Hz}, 1 \mathrm{H})$, $5.92(\mathrm{~d}, J=1.2 \mathrm{~Hz}, 1 \mathrm{H}), 6.54(\mathrm{~s}, 1 \mathrm{H}) 6.79(\mathrm{~s}, 1 \mathrm{H}),{ }^{13} \mathrm{C} \mathrm{NMR}\left(100 \mathrm{MHz}, \mathrm{CDCl}_{3}\right)$ $\delta 29.71,39.57,46.68,51.77,53.40,71.61,87.00,100.89,105.96,108.84,128.33$, 130.29, 146.08, 146.55, 170.76; LR MS (EI) m/z $273\left(\mathrm{M}^{+}\right)$, 200, 185; HR MS (EI) calcd for $\mathrm{C}_{15} \mathrm{H}_{15} \mathrm{NO}_{4} 273.1001$, found 273.1009.

\section{(6-Allyl-5-ethynyl-5,6,7,8-tetrahydro-[1,3]dioxolo[4,5-g]isoquinolin-5-yl)-acetic}

acid methyl este (3f). To a suspension of $\mathbf{3 f}(100 \mathrm{mg}, 0.366 \mathrm{mmol})$ and $\mathrm{K}_{2} \mathrm{CO}_{3}(253$ $\mathrm{mg}, 1.83 \mathrm{mmol}, 5$ equiv.) in $\mathrm{CH}_{3} \mathrm{CN}(1.2 \mathrm{~mL})$ was added ally bromide $(0.12 \mathrm{~mL}, 1.46$ mmol, 4 equiv.) and the solution was stirred at room temperature for $60 \mathrm{~h}$. Water was added and the aqueous layer was extracted with ethyl acetate. The organic layer was washed with brine, dried over $\mathrm{Na}_{2} \mathrm{SO}_{4}$ and concentrated to give $11(116.4 \mathrm{mg})$. 


\section{Acetic acid 1-(6-allyl-5-ethynyl-5,6,7,8-tetrahydro-[1,3]dioxolo[4,5-g]isoquinolin-}

5-ylmethyl)-allyl ester (13). A solution of 11 (116.4 mg) in THF was added $\mathrm{LiAlH}_{4}$ (36 mg, $0.95 \mathrm{mmol}$, 3equiv.) at $-78^{\circ} \mathrm{C}$ and the solution was stirred at $0{ }^{\circ} \mathrm{C}$ for $2 \mathrm{~h}$. To this suspension was added $\mathrm{Na}_{2} \mathrm{SO}_{4} \cdot 10 \mathrm{H}_{2} \mathrm{O}$ and the suspension was stirred at room temperature overnight. An undissolved material was filtered off and the filtrate was concentrated to give alcohol $(102.7 \mathrm{mg})$. To the solution of oxalyl chloride $(0.1 \mathrm{ml}$, $1.1 \mathrm{mmol}$, 3equiv.) in $\mathrm{CH}_{2} \mathrm{Cl}_{2}(1.5 \mathrm{~mL})$ was added DMSO $(0.16 \mathrm{~mL}, 2.2 \mathrm{mmol}, 6$ equiv.) at $-78{ }^{\circ} \mathrm{C}$ and the solution was stirred for $2 \mathrm{~min}$. A solution of $12(102.7 \mathrm{mg})$ in $\mathrm{CH}_{2} \mathrm{Cl}_{2}(1.5 \mathrm{~mL})$ was stirred at $-78{ }^{\circ} \mathrm{C}$ for $30 \mathrm{~min}$. To this solution was added $\mathrm{NEt}_{3}$ $(0.6 \mathrm{~mL})$. After the solution was stirred at $0{ }^{\circ} \mathrm{C}$ for $30 \mathrm{~min}$, water was added. The aqueous layer was made basic by $\mathrm{K}_{2} \mathrm{CO}_{3}$ and extracted with ethyl acetate. The organic layer was washed with brine dried over $\mathrm{Na}_{2} \mathrm{SO}_{4}$ and concentrated. The crude aldehyde was dissolved in THF ( $2 \mathrm{~mL})$ and to this solution was added vinyl magnesium bromide (THF solution, $1.0 \mathrm{M}, 1.1 \mathrm{~mL}, 1.1 \mathrm{mmol}$ ) at $-78{ }^{\circ} \mathrm{C}$. After the solution was stirred for $2 \mathrm{~h}$ at the same temperature, water was added. The aqueous layer was extracted with ethyl acetate and the organic layer was washed with brine dried over $\mathrm{Na}_{2} \mathrm{SO}_{4}$ and concentrated. The residue $12(102 \mathrm{mg})$ was dissolved in pyridine $(1 \mathrm{~mL})$. To this solution was added $\mathrm{Ac}_{2} \mathrm{O}(0.5 \mathrm{~mL}, 5.3 \mathrm{mmol})$ and DMAP $(5$ $\mathrm{mg}$ ) and the solution was stirred at room temperature for $14 \mathrm{~h}$. Water was added and the aqueous layer was extracted with ethyl acetate. The organic layer was washed with brine dried over $\mathrm{Na}_{2} \mathrm{SO}_{4}$ and concentrated. The residue was purified by column chromatography on silica gel (hexane/ethyl acetate, 4/1) to give colorless oil of $\mathbf{1 3}$ (90.2 mg, 70\% from 3f). less polar: ${ }^{1} \mathrm{H} \mathrm{NMR}\left(400 \mathrm{MHz}, \mathrm{CDCl}_{3}\right) \delta 2.01(\mathrm{~s}, 3 \mathrm{H})$, $2.29(\mathrm{dd}, J=15.4,5.4 \mathrm{~Hz}, 1 \mathrm{H}), 2.46(\mathrm{~s}, 1 \mathrm{H}), 2.46-2.51(\mathrm{~m}, 2 \mathrm{H}), 2.65(\mathrm{dd}, J=15.4$, $5.4 \mathrm{~Hz}, 1 \mathrm{H}), 2.77-2.97(\mathrm{~m}, 3 \mathrm{H}), 3.64(\mathrm{~d}, J=14.0 \mathrm{~Hz}, 1 \mathrm{H}), 4.83(\mathrm{~d}, J=10.4 \mathrm{~Hz}, 1$ H), $4.92(\mathrm{~d}, J=16.8 \mathrm{~Hz}, 1 \mathrm{H}), 5.16(\mathrm{~d}, J=10.4 \mathrm{~Hz}, 1 \mathrm{H}), 5.23-5.27(\mathrm{~m}, 2 \mathrm{H})$, 5.39 (ddd, $J=17.2,10.4,6.2 \mathrm{~Hz}, 1 \mathrm{H}), 5.82-5.96$ (m, $1 \mathrm{H}), 5.91$ (d, $J=11.6 \mathrm{~Hz}, 2 \mathrm{H})$, 6.49 (s, $1 \mathrm{H}), 6.82\left(\mathrm{~s}, 1 \mathrm{H}\right.$; ${ }^{13} \mathrm{C} \mathrm{NMR}\left(100 \mathrm{MHz}, \mathrm{CDCl}_{3}\right) \delta 21.51,29.81,43.96$, 
$44.79,53.91,60.11,71.47,73.34,84.44,100.86,107.28,108.07,114.93,116.75$, 129.81, 130.72, 136.40, 136.47, 146.11, 146.35, 169.61. more polar: ${ }^{1} \mathrm{H}$ NMR (400 $\left.\mathrm{MHz}, \mathrm{CDCl}_{3}\right) \delta 1.57(\mathrm{~s}, 3 \mathrm{H}), 2.35-2.57(\mathrm{~m}, 3 \mathrm{H}), 2.46(\mathrm{~s}, 1 \mathrm{H}), 2.53(\mathrm{dd}, J=11.2$, $11.2 \mathrm{~Hz}, 1 \mathrm{H}), 2.82-2.92$ (m, $2 \mathrm{H}), 2.98$ (d, $J=11.2 \mathrm{~Hz}, 1 \mathrm{H}), 3.51(\mathrm{~d}, J=14.8 \mathrm{~Hz}, 1$ H), $5.04(\mathrm{~d}, J=10.0 \mathrm{~Hz}, 1 \mathrm{H}), 5.08(\mathrm{~d}, J=15.6 \mathrm{~Hz}, 1 \mathrm{H}), 5.17(\mathrm{~d}, J=10.0 \mathrm{~Hz}, 1 \mathrm{H})$, $5.32(\mathrm{~d}, J=16.8 \mathrm{~Hz}, 1 \mathrm{H}), 5.65(\mathrm{~d}, J=6.4 \mathrm{~Hz}, 1 \mathrm{H}), 5.73(\mathrm{ddd}, J=16.8,10.0,5.6 \mathrm{~Hz}$, $1 \mathrm{H}), 5.80-5.91(\mathrm{~m}, 1 \mathrm{H}), 5.88(\mathrm{~s}, 1 \mathrm{H}), 5.91(\mathrm{~s}, 1 \mathrm{H}), 6.47(\mathrm{~s}, 1 \mathrm{H}) 6.91(\mathrm{~s}, 1 \mathrm{H}),{ }^{13} \mathrm{C}$ NMR (100 MHz, $\left.\mathrm{CDCl}_{3}\right) \delta 20.46,29.80,44.51,44.74,54.03,59.66,70.00,73.00$, $84.84,100.80,107.91,108.24,114.92,116.61,129.08,130.62,136.14,137.08$, $145.74,146.03,169.35$.

Ring Closure Metathesis of 13. To a solution of $\mathbf{1 3}(8.2 \mathrm{mg}, 0.023 \mathrm{mmol})$ in ether (1 $\mathrm{ml}$ ) was added $\mathrm{HCl}$ solution in ether $(1.0 \mathrm{M}, 0.05 \mathrm{~mL}, 0.05 \mathrm{mmol}$, 3equiv.) and the solvent was removed under reduced pressure. A solution of the residue and $\mathbf{1 6}$ ( $2 \mathrm{mg}$, $0.002 \mathrm{mmol}, 10 \mathrm{~mol} \%)$ was dissolved in $\mathrm{CH}_{2} \mathrm{Cl}_{2}(0.5 \mathrm{~mL})$. The solution was stirred at room temperarure under argon for $16 \mathrm{~h}$. The $\mathrm{CH}_{2} \mathrm{Cl}_{2}$ solution was washed with aqueous $\mathrm{K}_{2} \mathrm{CO}_{3}$ solution, and brine, dried over $\mathrm{Na}_{2} \mathrm{SO}_{4}$ and concentrated. The residue was purified by preparative chromatography on silica gel (ethyl acetate/ $\mathrm{MeOH}, 5 / 1$ ) to give $\mathbf{1 4 a}(4.5 \mathrm{mg}, 50 \%)$ and $\mathbf{1 4 b}(4.5 \mathrm{mg}, 50 \%)$.

\section{Erythrocarine 6b}

A solution of 14a $(4.5 \mathrm{mg}, 0.015 \mathrm{mmol})$ and $\mathrm{K}_{2} \mathrm{CO}_{3}(3.4 \mathrm{mg}, 0.025 \mathrm{mmol})$ in $\mathrm{MeOH}$ $(0.5 \mathrm{ml})$ was stirred at $0{ }^{\circ} \mathrm{C}$ for $1 \mathrm{~h}$. Water was added and the organic layer was extracted with ethyl acetate. The organic layer was washed with brine, dried over $\mathrm{Na}_{2} \mathrm{SO}_{4}$ and concentrated. The residue was purified by preparative chromatography on silica gel (ethyl acetate/ $\mathrm{MeOH}, 5 / 1)$ to give erythrocarine $\mathbf{6 b}(3.7 \mathrm{mg}, 93 \%)$.

In a similar manner, $14 \mathrm{~b}(4.5 \mathrm{mg})$ was treated with $\mathrm{K}_{2} \mathrm{CO}_{3}$ in $\mathrm{MeOH}$ for $4 \mathrm{~h}$ to give 15b (2.0 mg, 53\%). Erythrocaline (6b): ${ }^{1} \mathrm{H} \mathrm{NMR}\left(400 \mathrm{MHz}, \mathrm{CDCl}_{3}\right) \delta 1.70(\mathrm{~s}, 3 \mathrm{H})$, 
2.39 (br, $1 \mathrm{H}), 2.55$ (d, $J=14.4 \mathrm{~Hz}, 1 \mathrm{H}), 2.79-2.91(\mathrm{~m}, 3 \mathrm{H}), 3.41(\mathrm{~d}, J=14.4 \mathrm{~Hz}, 1$ H), $3.62(\mathrm{~m}, 1 \mathrm{H}), 3.86(\mathrm{br}, 1 \mathrm{H}), 5.38(\mathrm{t}, J=5.6 \mathrm{~Hz}, 1 \mathrm{H}), 5.87(\mathrm{~s}, 1 \mathrm{H}), 5.88(\mathrm{~s}, 1 \mathrm{H})$, $5.92(\mathrm{~s}, 1 \mathrm{H}), 5.99-6.05(\mathrm{~m}, 1 \mathrm{H}), 6.61(\mathrm{~s}, 1 \mathrm{H}), 6.77(\mathrm{~d}, J=10.0 \mathrm{~Hz}, 1 \mathrm{H}), 6.88(\mathrm{~s}, 1$ H). Epierythrocaline (15b): ${ }^{1} \mathrm{H}$ NMR $\left(400 \mathrm{MHz}, \mathrm{CDCl}_{3}\right) \delta 3.50(\mathrm{~d}, J=10.0 \mathrm{~Hz}, 1$ H), $3.74(\mathrm{~d}, J=15.0 \mathrm{~Hz}, 1 \mathrm{H}), 5.43(\mathrm{br}, 1 \mathrm{H}), 5.77$ (s, $1 \mathrm{H}), 5.82(\mathrm{dd}, J=10.0,2.4 \mathrm{~Hz}$, $1 \mathrm{H}), 5.88$ (s, $2 \mathrm{H}), 6.58(\mathrm{dd}, J=10.0,2.4 \mathrm{~Hz}, 1 \mathrm{H}), 6.60$ (s, $1 \mathrm{H}) .6 .78(\mathrm{~s}, 1 \mathrm{H})$.

\section{References}

1) Schunn, R. A. Inorg. Synth. 1974, 15, 5. 